\title{
A Novel Ensemble Wind Speed Forecasting Model in the Longdong Area of Loess Plateau in China
}

\author{
Tonglin Fu $\mathbb{D}^{1}$ and Chen Wang $\mathbb{D}^{2}$ \\ ${ }^{1}$ School of Mathematics \& Statistics, Longdong University, Qingyang, Gansu 745000, China \\ ${ }^{2}$ School of Mathematics \& Statistics, Lanzhou University, Lanzhou 730000, China \\ Correspondence should be addressed to Tonglin Fu; futonglin2008@163.com and Chen Wang; chenwang15@lzu.edu.cn
}

Received 12 July 2018; Accepted 19 November 2018; Published 10 December 2018

Academic Editor: Isabel S. Jesus

Copyright (c) 2018 Tonglin Fu and Chen Wang. This is an open access article distributed under the Creative Commons Attribution License, which permits unrestricted use, distribution, and reproduction in any medium, provided the original work is properly cited.

Energy is the lifeline and the base of development of social economy in every country or area. However, with the increasing depletion of fossil energy, it is imperative for business managers and government policy makers to seek and utilize new energy resources for keeping the national economy development sustained, rapid, and healthy. As a new energy resource, wind energy is developing with a high speed in the world for the reasons that it is an abundant natural resource with the characteristics of renewable, low cost, and no pollution. But in reality, forecasting wind speed accurately is continuously a challenging subject in the field of the wind energy development for a long time. In this paper, a novel ensemble wind speed forecasting model based on machine leaning models and hybrid models with data preprocessing technology is proposed to forecast the wind speed effectively in the Longdong area of Loess Plateau in China; numerical results show that the ensemble model has better precision, adaptability, and robustness.

\section{Introduction}

The modernization of the world economy is benefiting from fossil fuels, such as oil, natural gas, coal, and nuclear fission in a wide range of applications. However, with the increasing gradually energy consumption and approaching depletion of traditional fossil fuel, the world is facing energy crisis. In the latter 20th century, there occurred three oil crises in the world, which inflicts a great impact on the world economy. Therefore, the development of new energy is imperative. In recent years, renewable energy (such as wind, solar, geothermal, biomass, tidal, and hydropower) has received increasing attention. As one of the environmentally friendly and reproducibility energy resources, wind energy, which is generated by atmospheric motions, becomes an important renewable and sustainable new resource. The rapid development of wind energy conversion technology has made wind energy become an important part of the strategy of sustainable development in many countries. The global wind power industry installed another $63,013 \mathrm{MW}$, an increase of $22 \%$ in 2015. Among them, the Chinese wind power installed 30,500MW, accounted for $28.4 \%$ of global wind power installation capacity [1].
Wind speed directly influences the generation of wind power and wind speed forecasting plays a decisive role in the wind energy domain; it is distinctly important that predicting the wind speed accurately is very important for farm owners such as power grid operation scheduling and also crucial for the control, maintenance, and resource planning of wind energy conversion systems and just only predicts accurately the wind speed of wind farm to prevent effectively the adverse effects and increase competitiveness in the electricity market. Accordingly, many significant studies have been devoted to developing efficient forecasts for wind speed such as conventional statistical models and machine learning models [2-7]. The most commonly used traditional statistical model is the autoregressive integrated moving average (ARIMA) model and the generalized autoregressive conditional heteroskedasticity (GARCH) that uses historical data to establish a forecasting model, which can capture the linear relationship of the time series dataset well. Cadenasa and Rivera [8] forecasted the short-term wind speed in the region of La Venta, Oaxaca, Mexico, by using the technique of ANN. Guo et al. [9] forecasted the long-term wind speed in the Zhangye Area of China by using the ARMA 
and GARCH forecasting models based on the first definite season index method. The forecasting errors are analyzed and compared with the ones obtained from the ARMA and GARCH model. The conventional statistical model uses historical data to establish a forecasting model, which can capture the linear relationship of the training dataset well, but the prediction precision of statistical model is lower owing to the stochastic nature and intrinsic complexity of wind speed. With the development of artificial techniques, artificial intelligence models such as BPNN, SVM, and RBF have been proposed to develop forecasting wind speed models $[6,10,11]$. In fact, wind speed prediction is a realistic and challenging issue. Wind exists because the sun unevenly heats the surface of the earth's surface. Thus, the wind speed may be closely related to other meteorological parameters such as temperature, barometric pressure, airflow, and humidity, and it also could be easily influenced by the landform and geomorphology parameters. Due to the chaotic and intrinsic complexity of weather parameters, no single method or model can perform well in the forecasting process. In order to improve prediction accuracy, hybrid models based on conventional statistical models combined with artificial intelligent models being proposed. Shukur and Lee [10] developed a hybrid model of Kalman filter (KF) and artificial neural network (ANN) based on ARIMA using daily wind speed data from Iraq and Malaysia. The effectiveness of ARIMA was applied to determine the inputs structure of KF and artificial neural network (ANN). Hu et al. [11] constructed a hybrid forecasting model, which combines the ensemble empirical mode decomposition (EEMD) and the support vector machine (SVM), to improve the quality of wind speed forecasting; the mean monthly wind speed of three wind farms located in Northwest China is forecasted by the proposed hybrid method. Guo et al. [12] proposed a hybrid seasonal autoregression integrated moving average and least square support vector machine (SARIMA-LSSVM) model to predict the mean monthly wind speed in Hexi Corridor of China. Wang et al. [13] developed a novel approach named WTT-SAM-RBFNN for short-term wind speed forecasting by applying wavelet transform (WT) technique into hybrid model which hybrids the seasonal adjustment method (SAM) and the RBFNN; the wind speed in Northwest China is used to evaluate the forecasting accuracy of the proposed approach. Jing Shi et al. [14] proposed two hybrid models, namely, ARIMA-ANN and ARIMA-SVM, to forecast the wind speed and power generation in USA; the forecasting performance is compared with the single ARIMA, ANN, and SVM forecasting models. At present, the combination approaches are widely adopted to improve forecasting accuracies and many researchers have devoted themselves to the study of combination forecasts. There are numerous articles discussing combining different models and the application of them, at the same time, some parameter selection and optimization technique combined with data processing strategy are adopted such as weightingbased combined approaches, data preprocessing technique combined approaches, parameter selection and optimization technique combined approaches, and data postprocessing technique combined approaches [15]. Zhao W. et al. [16] proposed a time varying weight combining method to predict the monthly electricity consumption in China. Xiao et al. [17] constructed two combination models, the nonegative constraint theory (NNCT) combination model and the artificial intelligence algorithm combination model to forecast the wind speed in Chengde region of China. At the same time, they reviewed the combined models for wind speed predictions and classified the combined wind speed forecasting approaches.

In summary, the combination forecasts are grouped into the constant weight combination forecast method or the variable weight combination forecast method [18-21]. Since the precision of wind speed predict model is closely correlated with the stabilization of the wind speed time series and the traditional weighting-based combined approaches, the classical single models have little effect on the suppression of the noise and the predictions results may not be very exact.

In reality, with the wind speed that is often nonlinear, irregular, and nonstationary, current forecasting models based on combining the single models or hybrid models based on data preprocessing technique could not adapt to various time series data. However, these models are not able to take care of both the importance of the model parameter optimization and the data preprocessing technology, which lead to poor forecasting accuracy. In this paper, to deal with the highly noisy and intrinsic complexity of the wind speed time series, single models (BP neural network (BPNN), Support Vector Machine (SVM), and Extreme Learning Machine (ELM)) and hybrid models based on ensemble empirical mode decomposition method (EEMD) and BPNN (EEMD-BPNN), EEMD-SVM, and EEMD-ELM are used to forecast the daily average wind speed one year ahead. The forecasting model of BPNN, SVM, ELM, EEMD-BPNN, EEMD-SVM, and EEMD-ELM is combined with the traditional combination method based on the proposed no-negative constraint theory (NNCT), and the genetic algorithm (GA) for weights based on NNCT (GA-NNCT) is used to determine the submodels weights. The proposed decomposition ensemble forecasting model is used to predict the daily average wind speed and compared with the model of BPNN, SVM, ELM, EEMDBPNN, EEMD-SVM, EEMD-ELM, and TCM. The goal of this document is to provide a novel decomposition ensemble forecasting model with higher wind speed forecasting accuracy in the Longdong area of Loess Plateau in China.

The rest of this paper is organized as follows: EEMD method is briefly described in Section 2 and BPNN, SVM, ELM, BPNN, SVM, and ELM are briefly introduced in Section 3. In Section 4, the main method of hybrid models EEMD-BPNN, EEMD-SVM, and EEMD-ELM is presented. The GA-NNCT model for wind speed forecasting is proposed in Section 5. In Section 6, the unique geographical location and climate characteristics of the study area and the proposed ensemble approaches are briefly introduced. The three experimental simulations and the effectiveness of the proposed methodology are discussed in Section 7. Finally, Section 8 concludes the researches of this study. 


\section{Data Preprocessing Technology}

In reality, the wind speed time series are nonstationary, irregular, and highly noisy due to the fact that wind speeds are influenced by a variety of factors. Predicting wind speed with the time series directly often has large error; this weakness can be counterbalance by using EEMD.

EEMD is an empirical, intuitive, direct, and self-adaptive data processing method which is extended from EMD to overcome the intrinsic drawback of mode mixing; it is suitable in decomposing nonlinear and nonstationary signal sequences [22, 23]. The main purpose of EEMD is to extract the intrinsic mode functions (IMFs) from the original time series. IMFs must satisfy the following conditions: (1) in each whole function, the number of maxima and minima and number of zero crossings must be equal or different at the most by one and (2) the functions must be symmetric with respect to local zero mean. With the above definition for the IMF, the function can be decomposed as follows $[22,23]$ :

(1) Find out all the maximum points of the original signal $x(t)$ and fit the upper envelope line of the original signal through the cubic spline function.

(2) Calculate the mean value of upper and lower envelope line represented as $m_{1}(t)$ and the first intrinsic mode function (IMF) is calculated as

$$
h_{1}(t)=x(t)-m_{1}(t)
$$

(3) Repeat the above process for $k$ times for $h_{1}(t)$ until $h_{1}(t)$ accords with the requirement of IMF that the mean value tends to be zero; that is,

$$
h_{k}(t)=h_{k-1}(t)-m_{k}(t) .
$$

The first component of IMF $f_{1}(t)=h_{k}(t)$ signifies the component with the highest frequency of signal $x(t)$.

(4) Separate $f_{1}(t)$ from $x(t)$ and the residual signal without the component of high frequency $f_{1}(t)$ is denoted as

$$
r_{1}(t)=x(t)-f_{1}(t)
$$

where $r_{1}(t)$ is regarded as the original signal and repeat the first three steps; the second component of IMF $f_{1}(t)$ is obtained. Repeat $n$ times and $n$ IMFs are gained.

$$
\begin{gathered}
r_{2}(t)=r_{1}(t)-f_{2}(t), \\
r_{3}(t)=r_{2}(t)-f_{3}(t), \\
\vdots \\
r_{n}(t)=r_{n-1}(t)-f_{n}(t) .
\end{gathered}
$$

When the termination condition is achieved, the repeat stops. The original signal $x(t)$ can be repressed as

$$
x(t)=\sum_{i=1}^{n} f_{1}(t)+r_{n}(t)
$$

where $r_{n}(t)$ is the residual function representing the average trend of signal. Each component of IMF $f_{1}(t), f_{2}(t), \cdots, f_{n}(t)$ separately includes the components of signal with different time scale features. EEMD defines the true IMF components as the mean of an ensemble of trials and each trial consists of the decomposition results of the signal plus a white noise of finite amplitude. The effect of the added white noise can be controlled by $\varepsilon_{n e}=\varepsilon / \sqrt{N E}$, where $N E$ is the number of ensemble members, $\varepsilon$ is the amplitude of the added noise, and $\varepsilon_{n e}$ is the final standard deviation of error, defined as the difference between the input signal and the corresponding IMFs.

In this paper, the algorithm of EEMD consisted of the following steps.

Step 1. Add a white noise series to the wind speed time series.

Step 2. Decompose the wind speed time series with added white noise into IMFs using the EMD algorithm.

Step 3. Repeat Step 1 and Step 1 iteratively with different white noise each time.

Step 4. The IMFs are obtained as the final results.

\section{Individual Forecasting Models}

The BPNN, SVM, and ELM algorithm are a class of effectively artificial intelligence algorithm, used extensively in some practical application fields including function approximation, prediction and automatic control, optimization, and pattern classification $[9,10,24,25]$. These methods have high precision in terms of either simulation or the result of the prediction with satisfactory results. In this paper, BPNN, SVM, and ELM are used to forecast the daily wind speed, respectively. The principle of BPNN, SVM, and ELM is briefly presented as follows.

3.1. Backpropagation Neural Network (BPNN). The BPNN has been widely used in linear and nonlinear regressions for data fitting and estimating model parameters, especially in uncovering nonlinearity between inputs and the outputs, even in the absence of sufficient information about the relationship between them [13]. The goal of any training algorithm is to minimize the global mean sum squared error $E$ between the real network output $z_{l}$ and the desired output $t_{l}$, defined as

$$
E=\frac{1}{2} \sum_{l}\left(t_{l}-z_{l}\right)^{2}
$$

where

$$
z_{l}=f\left(\sum_{j} v_{l j} \varphi\left(\sum_{j} w_{j i} x_{i}-\theta_{j}\right)-\theta_{l}\right),
$$

$w_{j i}$ is the weight of the connection from input $i$ to neuron $j$, $v_{l j}$ is the weight of the connection from neuron $j$ to output $l, x_{i}$ is input signal, $\theta_{l}, \theta_{j}$ are the threshold, and $f(\cdot), \varphi(\cdot)$ are activation function. Typical activation functions of BPNN are logistic sigmoid function, hyperbolic tangent sigmoid 
function, and linear function. In this paper, the activation function

$$
f(x)=\frac{2}{1+\exp (-2 x)}-1 .
$$

To achieve better prediction results, we use the Hecht-Nelson method to determine the node number of the hidden layer: when the node number of the input layer is $n$, the node number of the hidden layer is $2 n+1$, with $n$ input neurons, $2 n+1$ hidden neurons and one output neuron. To ensure the quality of forecast results, we adopt normalized method to treat the input and output data in advance of training the network; the formula is

$$
\mathrm{x}^{\prime}=\left\{x_{i}^{\prime}\right\}=\frac{x_{i}-x_{i \min }}{x_{i \max }-x_{i \min }}, \quad i=1,2, \cdots, n .
$$

In this paper, the logistic sigmoid function is adopted as activation function and the node number of the hidden layer is determined by the Hecht-Nelson method. The input and output data are normalized in advance of training the network to ensure the quality of forecast results.

3.2. Support Vector Machine (SVM). SVM is widely used to solve classification recognition and forecasting problems in terms of the limited sample sizes. The main idea of SVM for regression is to map the data into a high-dimensional feature space via nonlinear mapping and to present a linear regression model in this feature space [4]. The regression formula is

$$
f(x)=\sum_{i=1}^{m} w_{i} \varphi_{i}(x)+b
$$

Based on the structural risk minimization principle, the coefficients $\left\{w_{i}\right\}, i=1,2, \cdots m$ can be obtained from the data by optimizing the following quadratic programming problem:

$$
\begin{array}{ll}
\min _{w, b, \zeta} & \frac{1}{2}\|w\|^{2}+C \sum_{i=1}^{n}\left(\xi_{i}+\xi_{i}^{*}\right) \\
\text { s.t. } & \left|y_{i}-\langle w \cdot \Phi(x)\rangle-b\right| \leq \varepsilon+\xi_{i}, \\
& \xi_{i} \geq 0, \quad \xi_{i}^{*} \geq 0, i=1,2, \cdots n
\end{array}
$$

where $\xi_{i}, \xi_{i}^{*}$ are slack variable and $\varepsilon$ is the predetermined maximum allowed deviation between the actual value and the estimated one. By solving the optimization problem, the estimation function can be obtained as follows:

$$
f\left(x, \alpha, \alpha^{*}\right)=\sum_{i=1}^{N}\left(\alpha_{i}-\alpha_{i}^{*}\right) k\left(x_{i}, x\right)+b
$$

with

$$
\sum_{i=1}^{N}\left(\alpha_{i}^{*}-\alpha_{i}\right)=0, \quad 0 \leq \alpha_{i}^{*}, \alpha \leq C,
$$

where $\alpha_{i}$ and $\alpha_{i}{ }^{*}, i=1,2, \cdots, n$ are Lagrange multipliers that can be obtained by solving the dual form of (11) and the kernel function $k\left(x_{i}, x\right)$ represents the inner product in the
D-dimensional feature space and meets Mercer's condition. In this paper, the kernel function

$$
k\left(x_{i}, x\right)=\exp \left\{-\frac{\left\|x-x_{i}\right\|^{2}}{2 \sigma^{2}}\right\} .
$$

3.3. Extreme Learning Machine (ELM). ELM is a singlehidden layer feed forward neural network; it accomplishes the learning process in two steps. First, the input weights and hidden biases are initialized with random numbers. Second, the output weights are calculated through an inverse operation on the hidden layer output matrix. The output function of ELM can be formulated as

$$
f(x)=\sum_{i=1}^{l} \alpha_{i} G\left(x ; w_{i}, b_{i}\right),
$$

where $\alpha=\left(\alpha_{1}, \alpha_{2}, \cdots, \alpha_{l}\right)$ is the output weight vector and $l$ is the number of hidden nodes, $w_{i}$ is the input weight vector that connects the $i$-th hidden node and input nodes, $b_{i}$ is the bias of the $i$-th hidden node, and $G$ is the activation function satisfying ELM universal approximation capability theorems; there are two kinds of hidden nodes that are usually employed in $\operatorname{ELM}[24,25]$. One is additive hidden nodes and the other is RBF hidden nodes. In this paper, the RBF is adopted as activation function, which can be formulated as

$$
G\left(x ; w_{i}, b_{i}\right)=G\left(\frac{\left\|x-w_{i}\right\|}{b_{i}}\right) .
$$

For a training set $\left(x_{k}, y_{k}\right)_{k=1}^{l}$, the output weight vector $\alpha$ can be determined by

$$
\widehat{\alpha}=H^{+} Y=\left(H^{T} H\right)^{-1} H^{T} Y,
$$

where

$$
H=\left(\begin{array}{ccc}
G\left(x_{1} ; w_{1}, \beta_{1}\right) & \cdots & G\left(x_{1} ; w_{l}, \beta_{l}\right) \\
\vdots & \cdots & \vdots \\
G\left(x_{m} ; w_{1}, \beta_{1}\right) & \cdots & G\left(x_{m} ; w_{l}, \beta_{l}\right)
\end{array}\right)
$$

and $\mathrm{H}^{+}$is the Moore-Penrose generalized inverse of $H$.

\section{Hybrid Models}

The BPNN, SVM, and ELM are useful methodologies and have been successfully applied to time series prediction with satisfactory prediction results in various fields $[3,4,6,8,9]$, but these models still have difficulties with higher wind speed forecasting accuracy. Furthermore, due to the characteristics of the highly noisy and intrinsic complexity of the original wind speed series, the single model cannot completely catch the characteristics of the data in a real problem. In order to improve the precision of wind speed forecasting effectively, there are a lot of hybrid models that have been proposed, and the accuracy of wind speed forecasts was effectively improved, such as the traditional combined approaches, data 
preprocessing method, parameter optimization technique, and data postprocessing algorithms $[13-20,26]$. In this paper, there are three hybrid forecasting models called EEMDBPNN, EEMD-SVM, and EEMD-ELM with the functions of decomposition, denoise, and forecasting modeling capabilities that are proposed.

The original wind speed time series is decomposed by EEMD into several independent intrinsic mode functions (IMFs) which are the various frequency subseries and one residual series. The IMF1 is regarded as white noise series, and it can be neglected since IMF1 is the most disordered and unsystematic part of the wind speed series; it sometimes has a great disturbance for the forecasting precision of wind speed, and then these simple subseries are separately modeled by BPNN, SVM, and ELM models, respectively, such that the tendencies of these subseries can be predicted. Finally, aggregating the prediction results of all subseries of BPNN, SVM, and ELM models, respectively, to produce three ensemble fitness and forecasting results for the original wind speed series, these models can be denoted by EEMD-BPNN, EEMD-SVM, and EEMD-ELM.

\section{Genetic Algorithms (GA) for Weights Based on No-Negative Constraint Theory (GA-NNCT)}

Many studies have shown that the forecasting risk of single model can be reduced and the forecasting accuracy can be improved by combining forecasts from conceptually different individual model $[11-15,17,20]$. Mathematically, the traditional combination model can be expressed as

$$
\mathrm{y}_{c, t}=\sum_{j=1}^{m} w_{j} y_{j, t}, \quad t=1,2, \cdots .
$$

where $y_{c, t}$ denote the combined output at time $t$ of the combining methods, $y_{j, t}$ denote the unbiased out-of-sample forecast for time series $x_{t}$ which is obtained by the $j t h$ individual model, $m$ is the number of the component models, and $w_{j}$ is the weight on the $j t h$ component model. These weights are all constrained to be $w_{j} \in[0,1]$ and have to meet the requirement:

$$
\sum_{j=1}^{m} w_{j}=1
$$

The best weight $w_{j}, j=1,2, \cdots m$ of the traditional combination method (TCM) can be obtained by solving the following quadratic programming problem:

$$
\begin{array}{ll}
\min & o b j=\sum_{t=1}^{T} e_{c, t}^{2}=\sum_{t=1}^{T} \sum_{j=1}^{m} \sum_{i=1}^{m} w_{i} w_{j} e_{j, t} e_{i, t}, \\
\text { s.t. } & \sum_{j=1}^{m} w_{j}=1, \\
& w_{j} \geq 0, \quad j=1,2, \cdots m .
\end{array}
$$

where $e_{j t}=x_{t}-y_{j, t}, t=1,2, \cdots T$ denote the residual of the $j$ th individual model at time $t$.
With this model the forecasting risk of single neural network can be reduced and the forecasting accuracy can be improved. The results of the traditional combination model indicate that if the forecasting accuracy of submodels is largely different or similar, the weight of the best forecast result would be assigned 1 and the worst would be assigned 0 . Such a theory seems very plausible, but, due to the characteristics of nonlinear, stochastic, and nonstationary wind speed, the forecasting performance of TCM is unsatisfied and could not further improve the prediction ability. To overcome the shortcomings of the TCM, the negative weights are assigned to the forecasting results of submodels, which is the central idea of no-negative constraint theory (NNCT). And the way it will work is that if the forecasting series trend is opposite that of the original series trend, a negative weight is assigned to the forecast; otherwise, a positive weight is assigned. The literature research numerical results show that the prediction effect is raised when negative weights are assigned [15, 17].

The genetic algorithm (GA) is a heuristic and stochastic optimization algorithm for solving optimization problems and nonlinearity mapping based on evolution theory and genetic principle [27]. Compared with classical methods, the GA has much superiority and practical value that quickly converges to the optimal solution by the genetic manipulations, which make GA in searching for the best weight in the ensemble forecasting model for wind speed forecasting. In this paper, the GA-NNCT are given as

$$
\begin{array}{ll}
\min & \text { obj }=\sum_{t=1}^{T} e_{c, t}^{2}=\sum_{t=1}^{T} \sum_{j=1}^{m} \sum_{i=1}^{m} w_{i} w_{j} e_{j, t} e_{i, t}, \\
\text { s.t. } & \sum_{j=1}^{m} w_{j} \approx 1, \\
& w_{j} \in[-1,1] .
\end{array}
$$

The best weights $w_{j}, j=1,2, \cdots m$ of GA-NNCT can be determined by using the GA, in which the encoding length can be determined by the number of combined models when the maximum iteration is 1000 , the chromosome population is 30 , the crossover probability $(p c)$ is 0.2 , and the mutation rate $(p m)$ is 0.1 . In addition, in this study the chromosomes of GA-NCT are encoded in $[0,1]$ and the chromosomes of GA-NNCT are encoded in $[-1,1]$. Selection chooses the better individual (weight) from the initial population that can be applied to the subsequent iteration according to the lowest fitness value. Crossover exchanges part of the chromosomes between a pair of parent individuals with the $p c$ and produces two new individuals (weights). Mutation generates new individuals by changing one or some gene values of the chromosomes according the $p m$.

\section{Study Area and Experimentation Design}

In this section, the unique geographical location and climate characteristics of the Longdong area of Loess Plateau in China area and construction situations about a series of wind power projects are introduced. The simulation processes 
TABLE 1: The numerical characters of the wind speed time series for study area.

\begin{tabular}{lccccccc}
\hline & Mean & Median & Variance & Skewness & Kurtosis & Minimum & Maximum \\
\hline Wind turbine I & 2.0366 & 1.9000 & 0.6302 & 1.1229 & 0.0427 & 0.3000 & 5.9000 \\
\hline Wind turbine II & 2.0093 & 2.0000 & 0.3840 & 1.0479 & 0.0526 & 0.3000 & 6.9000 \\
\hline Wind turbine III & 2.1484 & 2.0000 & 0.4757 & 0.9260 & 0.0432 & 0.7000 & 5.4000 \\
\hline
\end{tabular}

are designed and the evaluation criteria of the proposed ensemble forecasting models are introduced.

6.1. Study Area and Available Data. The Longdong area of Loess Plateau in China is $36^{\circ} 1^{\prime}-37^{\circ} 9^{\prime}$ latitude and $106^{\circ} 21^{\prime}$ $107^{\circ} 44^{\prime}$ Longitude. It is a part of the gully region along the midstream of the Yellow River in China. The flat top is less than $10 \%$ of the plateau. Its topography is mainly made of highland (9.24\%), hills and mountains (61.11\%), river beach $(2.38 \%)$, and valley slope (27.27\%). The plateau extends from the northwest to the southeast, the highest point is 2,089 meters altitude, and the lowest point is 885 meters altitude. The relative elevation is 1,204 meters, with a vertical slope of about $0.58 \%$. It is $207 \mathrm{~km}$ from the north to the south and $208 \mathrm{~km}$ from the east to the west, with average annual rain fall of about $300 \mathrm{~mm}$ and an annual average sunshine of 2,600 hours. It is a mountainous areas that lies in the convergence zone of the $\mathrm{Mu}$ Us Desert and Loess Plateau. Its special geographical features and long-term agricultural practices have caused serious soil erosion and degradation of the environment. Because of its special geographical environment and backward economic development, the Loess Plateau in Longdong area is classified as one of the key regions of the national poverty alleviation plan.

The climate of the Longdong area in Loess Plateau is temperate continental monsoon, which is suitable for the construction of wind power. With the state's call for the construction of new energy and poverty reduction, the local government adjusts the industrial structure, optimizes the allocation of resources, and develops new energy. In June 2011, the Loess Plateau in Longdong area started the construction of million kilowatt wind power base, planning a total installed capacity of about 100MW. The first phase of construction capacity is $49.5 \mathrm{MW}$ and $110 \mathrm{KV}$ booster station is built transforming the power from Tianshui fort substation to the national power grid. Since being put into operation in late June 2012, equipment is in good condition and grid connects power normally. In October 2012, it has been generating more than $1000 \mathrm{KWH}$. In November 2014, six wind power plants with a total installed capacity of 1,050 MW started construction. To the end of April of 2015, the cumulative generating of Maojing fort substation is 481million $\mathrm{KWH}$ and another installed capacity of 20MWwind farm has been started construction [28].

Therefore, in order to optimize network management, forecast wind strength, and electricity load and build bigger wind power plants, there is an urgent need for predicting wind speed. In this paper, three time series from three different fort substations in Longdong area of the Loess Plateau from 1 January, 2007, to 31 December, 2015, are selected to investigate the effectiveness and superiority of the proposed combination model. The dataset from 1 January, 2007, to 31 December, 2014, is used as the training set in the predicting procedure, and the set from 1 January, 2015, to 31 December, 2015, is used as the test set. Figure 1 shows the geographic location, topography, and morphology of study area and wind speed curves that are nonlinear, irregular, and nonstationary. Table 1 shows the numerical characters of the wind speed time series for wind turbine I, wind turbine II, and wind turbine III.

6.2. Experimentation Design and Evaluation Criteria. The wind speed dataset in Longdong area of the Loess Plateau was simulated by the proposed ensemble GA-NNCT forecasting models. The flowchart of ensemble approaches is shown in Figure 2 and the following steps show the simulation process.

Step 1. Three single models, BPNN, SVM, and ELM are used to forecast the daily wind speed; the forecasting accuracies of these three models are compared.

Step 2. EEMD is employed to decompose the original time series data into a number of independent IMFs and one residual series. The highest frequency bandIMF1 is discarded as white noisy signals to make preparations for next stage.

Step 3. The rest of IMFs are forecasted independently by using BPNN, SVM, and ELM; the predicted results are aggregated into an ensemble result, respectively. There are three hybrid models based on the data preprocessing techniques including EEMD-BPNN, EEMD-SVM, and EEMDELM that are constructed and the errors of the models are compared.

Step 4. The forecasting results of the BPNN, SVM, ELM, EEMD-BPNN, EEMD-SVM, and EEMD-ELM are selected and the GA-NNCT is used to optimize the weight of the component models, respectively; the daily wind speeds are predicted and the errors of the above models are compared.

In order to quantitatively determine the best model, the mean square error (MSE), mean absolute error (MAE), and mean absolute percentage error (MAPE) are used to evaluate the forecasting precision of the ensemble model; these measures are as follows:

$$
\begin{aligned}
\mathrm{MSE} & =\frac{1}{n} \sum_{i=1}^{n}\left(x_{i}-\widehat{x}_{i}\right)^{2} \\
\mathrm{MAE} & =\frac{1}{n} \sum_{i=1}^{n}\left|x_{i}-\widehat{x}_{i}\right|,
\end{aligned}
$$



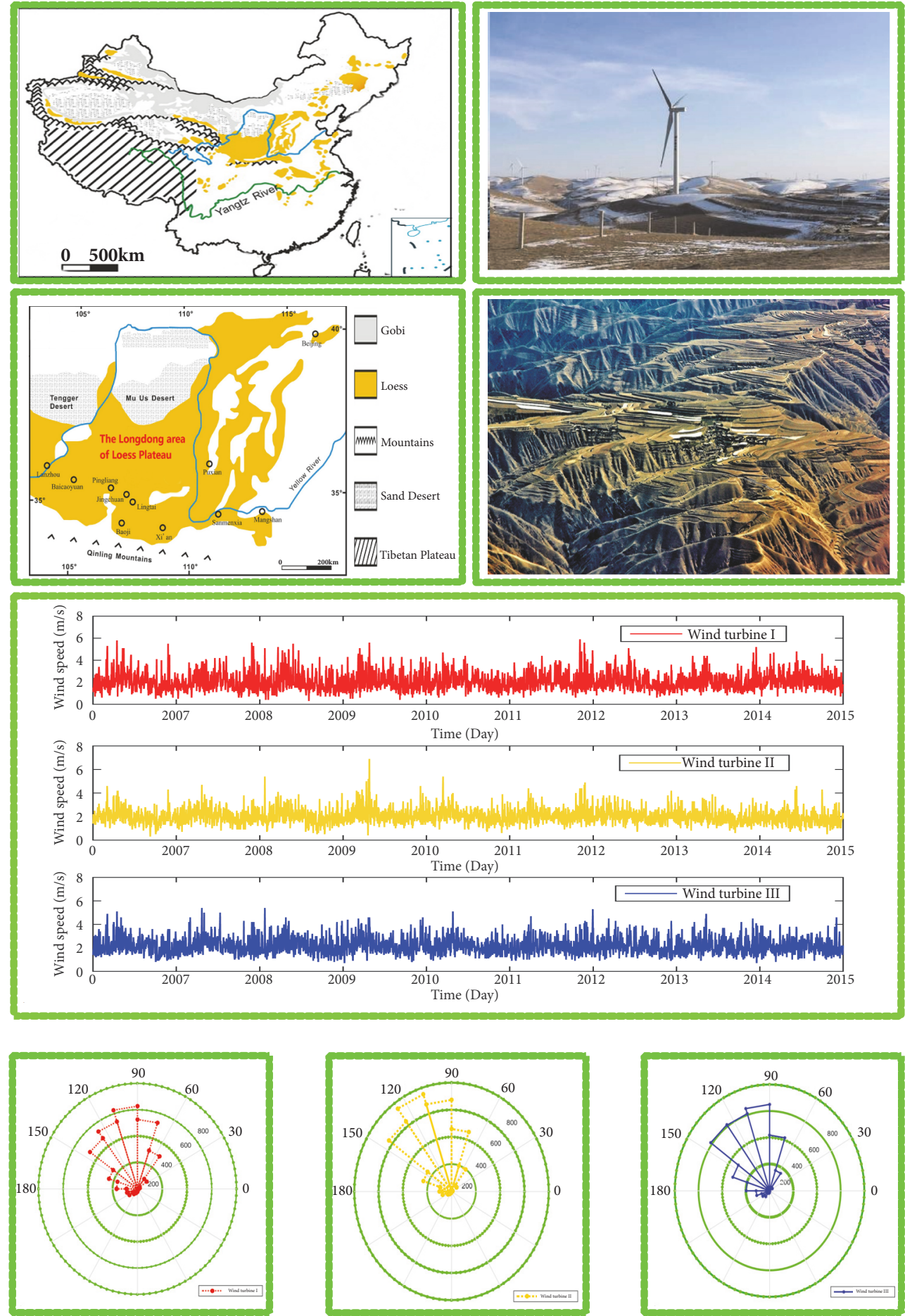

FIGURE 1: Daily average wind speed curves in the Longdong area of the Loess Plateau in China.

$$
\mathrm{MAPE}=\frac{1}{\mathrm{n}} \sum_{i=1}^{n}\left|\frac{x_{i}-\widehat{x}_{i}}{x_{i}}\right| \times 100 \%,
$$

where $x_{i}$ and $\hat{x}_{i}$ represent the $i t h$ actual and forecasted values, respectively, and $n$ is the sample size. The MSE and MAE reveal the average variance between the forecast value and the true value while the MAPE, as unit-free measure, has good sensitivity for small changes in data, does not display data asymmetry, and has very low outlier protection. The deviation between actual values and forecasting values are measured by those evaluation criteria; therefore, the smaller values of evaluation index mean the better performance of the model. If the results are not consistent with each other, the MAPE will be selected as the final benchmark. 


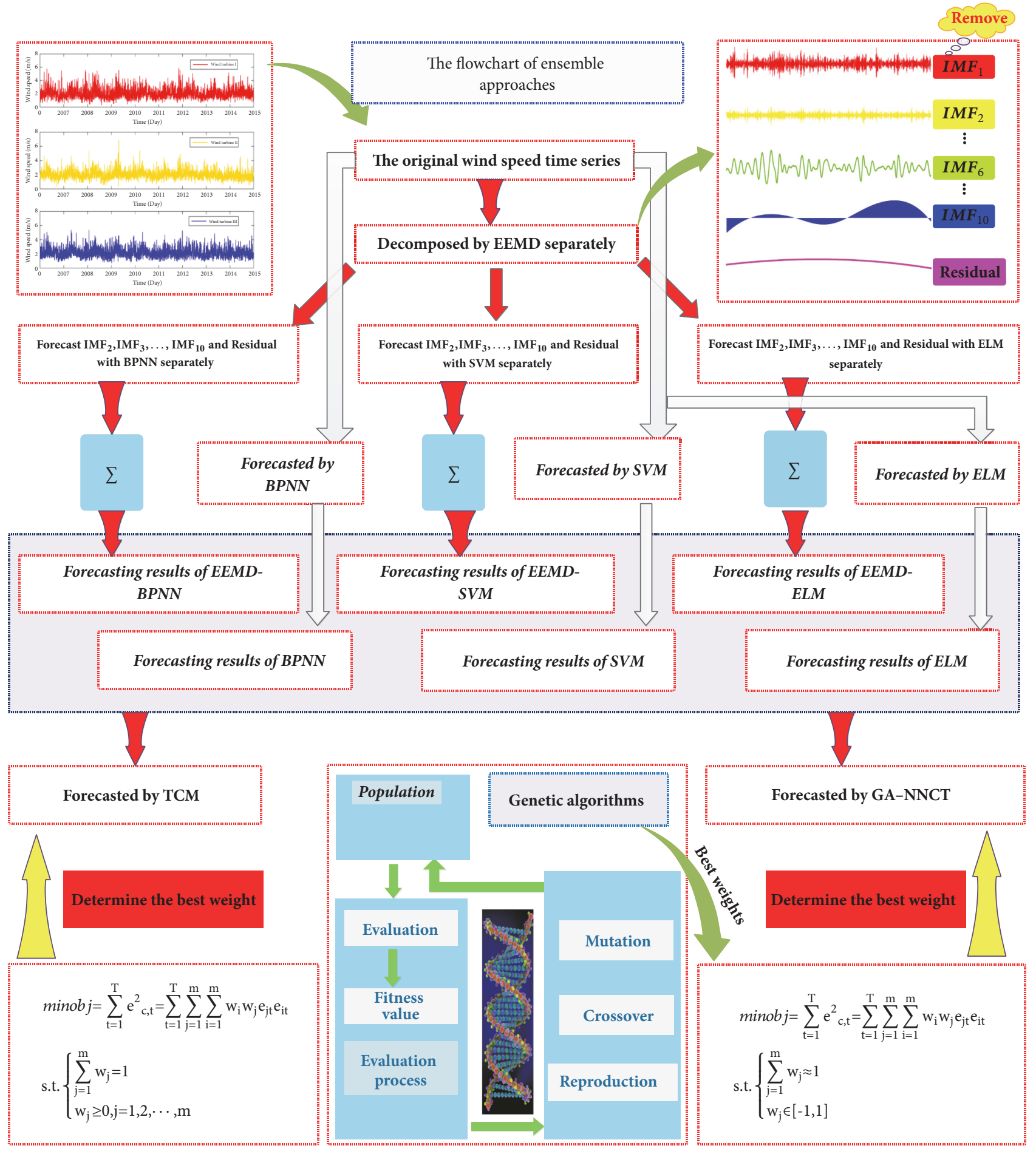

Figure 2: Flowchart for the ensemble approaches.

\section{Results and Discussions}

Wind speed is the essential factor which affects the wind power output and just only predicts accurately the wind speed of wind farm to prevent effectively the adverse effects and increase competitiveness in the electricity mark.

Compared with the traditional linear module, the machine leaning models have better precision and adaptability.
Although the machine learning algorithms eliminate the limitations of traditional regression methods and can forecast the daily wind speed with higher accuracy, it can be seen from Figure 2 that the wind speed time series are nonstationary and highly noisy due to the fact that wind speeds are affected by a variety of factors; predicting wind speed with the noisy data may be subject to large errors. 


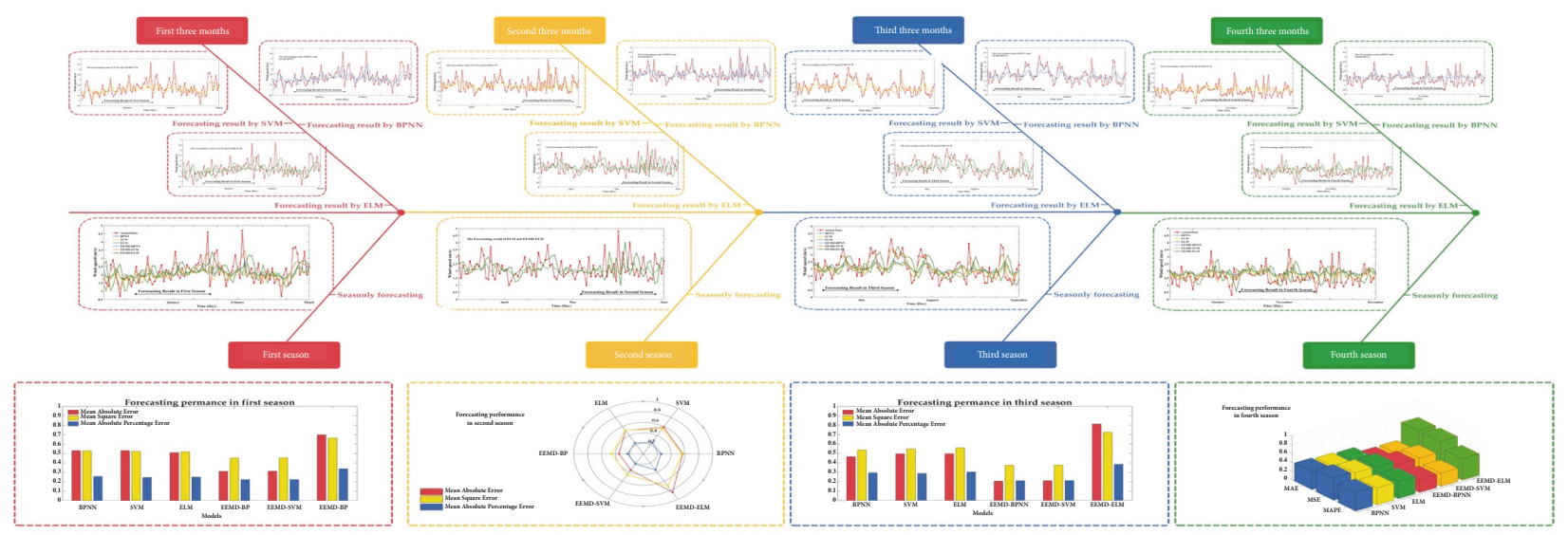

FIgURE 3: The forecasting performance of submodels in experiment I for wind turbine I.

In order to improve prediction accuracy, hybrid models are based on data preprocessing technique combined with artificial intelligent models being proposed and used in the wind speed forecasting field. In this study, the EEMD is used as a noise cancellation method and the daily average wind speed series are decomposed into several independent IMFs. IMF1 is neglected since it is the highest frequency band, which is regarded as white noise series. The BPNN, SVM, and ELM are used to forecast each independent IMFs and the prediction results of all subseries of BPNN, SVM, and ELM models are aggregated, respectively, to produce three forecasting results for the original wind speed series.

7.1. Experiment I: Daily Wind Speed Forecasted by the Submodels. Experiment I was designed to verify the effectiveness of the single machine leaning models and hybrid models using the wind turbine I dataset. If the hybrid models are adaptability and robustness, it can be concluded that the hybrid models EEMD-BPNN, EEMD-SVM, and EEMDELM are effective in forecasting the daily wind speed. The experiment processes are clearly shown in the following.

Step 1. There are three popular single machine learning models including BPNN, SVM, and ELM which are used to forecast the daily wind speed; the forecasting accuracies of these three models are compared.

Step 2. EEMD is employed to decompose the original data into a number of independent IMFs and one residual series. The highest frequency bandIMF1 is discarded as white noisy signals to make preparations for next stage.

Step 3. Three hybrid models including EEMD-BPNN, EEMD-SVM, and EEMD-ELM are used to forecast the daily average wind speed.

Step 4. The comparisons of forecasting accuracy for the mean daily wind speed in the wind turbine I of Longdong area of the Loess Plateau in China are made between the submodels BPNN, SVM, ELM, EMD-BPNN, EEMD-SVM, and EEMDELM.
Figure 3 shows the forecasting daily wind speed and the evaluation criteria of different submodels. In order to investigate these submodels predicting precision in different time scale, the evaluation criteria of MSE, MAE, and MAPE in each season are listed in Table 2. As shown in Table 2, the following main conclusion can be obtained:

(a) The forecasting accuracies of EEMD-BPNN, EEMDSVM, and EEMD-ELM are all higher than the BPNN, SVM, and ELM, respectively, which means that the hybrid models based on data preprocessing method have better forecasting performance than the corresponding single machine leaning model.

(b) The hybrid models prediction accuracy can be improved significantly based on data preprocessing technology and single machine leaning model, but none of forecasting models fits all application conditions properly.

As shown in Table 2, compared with BPNN, SVM, and ELM, the MAPE of EEMD-BPNN, EEMD-SVM, and EEMDELM decreased about $3.10 \%$ to $5.42 \%$ in the first threequarters of 2015, and, in the fourth season, the MAPE of EEMD-BPNN, EEMD-SVM, and EEMD-ELM decreased about $6.55 \%$ to $10.51 \%$, which indicate EEMD-BPNN, EEMDSVM, and EEMD-ELM with a better accuracy compared to BPNN, SVM, and ELM in each season. In different seasons, the results in Table 2 show that the model prediction accuracies of the hybrid models are a little different from each other. If the MAPE is selected as the evaluation criterion, the minimum MAPE of EEMD-BPNN, EEMD-SVM, and EEMD-ELM indicates that the corresponding hybrid model has the best performance for forecasting daily average wind speed in each season. In fourth season, the minimum MAPE of EEMD-BPNN, EEMD-SVM, and EEMD-ELM is 24.06\%, which means that the forecast stability of hybrid models with the nonstationary data need to be improved, although the data preprocessing technology has perfect effect on improving the forecasting accuracy. The evaluation criteria of MSE, MAE and MAPE of submodels in year 2015 are calculated and the results are shown in Table 7. Among single submodels, the 


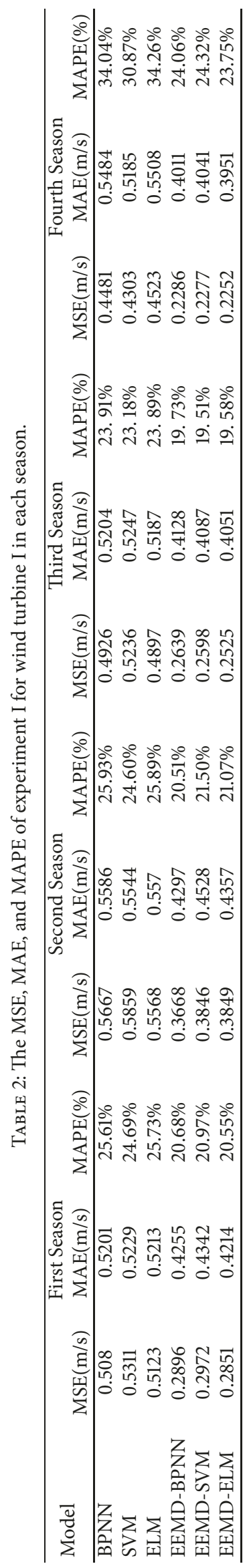


MAPE of BPNN, SVM, and ELM shows that the prediction precision of the SVM is the highest, ELM model is in the second place, and the BPNN is in the end. Comparing with single BPNN, SVM, and ELM, the forecasting accuracy of EEMD-BPNN, EEMD-SVM, and EEMD-ELM increased to $6.148 \%, 4.26 \%$, and $6.22 \%$, respectively. Overall, numerical results in Table 7 show the MAPE of EEMD-ELM is $21.24 \%$, which means that the submodel EEMD-ELM has excellent forecasting performance.

In summary, the single machine learning algorithm has its unique advantages in the wind speed prediction, but the forecasting accuracy needs to be improved. The data preprocessing technology is an efficient method in improving the forecasting accuracy. The forecasting performance is different in various time scales when comparing the forecasting performance of the forecast submodels BPNN, SVM, ELM, EEMD-BPNN, EEMD-SVM, and EEMD-ELM. In order to achieve a better forecast effect, it is important to choose different forecasting model for distinctive performance features or characteristics in different time scales. However, none of forecasting models fits all application conditions, which makes the integrated navigation technology much more important.

To demonstrate the forecasting performance of the proposed model in terms of forecasting accuracy, the Wilcoxon signed-rank test is implemented. The Wilcoxon signed-rank test is a nonparametric procedure employed in hypothesis testing situations and is used to compare the significant differences in terms of central tendency between two datasets with the same size $[29,30]$. Table 4 shows the results of Wilcoxon signed-rank test for wind turbine I; the levels of Wilcoxon signed-rank test are all smaller than the significance level 0.01 , which represents that the test indicates not to accept the null hypothesis and the data preprocessing technology has significant contribution in improving the forecasting accuracy.

7.2. Experiment II: Daily Wind Speed Forecasted by the TCM Model. The numerical results in Tables 2 and 7 show that individual forecasting model or the hybrid models are not ideal when they are applied to daily wind speed predicting in the Longdong area of the Loess Plateau as the forecasting trend has segmental instability to the original nonstationary time series. In order to reduce the forecasting risk of single model and improve the forecasting accuracy, the forecasting model of BPNN, SVM, ELM, EEMD-BPNN, EEMDSVM, and EEMD-ELM is combined with the TCM and the forecasting performance of TCM is compared with the model of BPNN, SVM, ELM, EEMD-BPNN, EEMD-SVM, and EEMD-ELM. According to formula (14), the best weight is assigned to submodels and the forecasting performance of TCM should be statistically significant, but, as shown in Table 7, the MAPE of TCM for wind turbine I is $21.68 \%$, which is higher than the EEMD-BPNN, EEMD-SVM, and EEMD-ELM that means the forecasting performance of TCM is no better than the hybrid models; this effect is utterly alien from the one we have intended. The results indicate that the prediction results of TCM are unsatisfied and could not further improve the prediction ability. In order to investigate whether this kind of circumstance is caused by the unique climate characteristics of wind turbine I or not, experiment II was designed by using wind turbine II dataset; simultaneously the numerical results can also be used to verify the adaptability and robustness of the ensemble TCM model. If the ensemble TCM model is adaptability and robustness, it can be concluded that the proposed approach has excellent performance in forecasting the wind speed. The experiment processes are demonstrated as follows.

Step 1. There are six submodels including BPNN, SVM, ELM EEMD-BPNN, EEMD-SVM, and EEMD-ELM that are used to forecast the wind speed using wind turbine II data.

Step 2. The forecasting results of submodels for wind turbine I and wind turbine II are combined by using TCM, respectively, and the error of the above models is compared.

The prediction results of TCM for wind turbine II are shown in Figure 4 and the MSE, MAE, and MAPE of experiment II for wind turbine II in each season are shown in Tables 3 and 7.

As shown in Tables 3 and 7, the following conclusion can be obtained.

(a) The forecasting accuracy of EEMD-BPNN, EEMDSVM, and EEMD-ELM for wind turbine II is all higher than the BPNN, SVM, and ELM in different time scales, respectively.

(b) The MAPE of TCM for wind turbine II are all smaller than the MAPE of BP, SVM, and ELM in different time scales, respectively, which means that the TCM have better performance than the single machine leaning models.

(c) Comparing with EEMD-BPNN, EEMD-SVM, and EEMD-SVM, the MAPE of TCM for wind turbine I and wind turbine II are not less than the MAPE of EEMDBPNN, EEMD-SVM, and EEMD-SVM in different time scales, which means that the forecasting performance of TCM has weak adaptability and robustness.

In summary, comparing with BPNN, SVM, and ELM, the EEMD-BPNN, EEMD-SVM, EEMD-ELM, and TCM model have higher forecasting precision for wind turbine I and wind turbine II. Although the data preprocessing technology (EEMD) and the combination method have obviously effect on improving the forecasting accuracy, the forecasting adaptability and robustness of EEMD-BPNN, EEMD-SVM, EEMD-ELM, and TCM model with the nonstationary data need to be improved. The adaptability of TCM, especially, is not ideal, although in some cases it may be acceptable.

Similar to experiment I, the Wilcoxon signed-rank test is implemented to demonstrate the significant contribution of the proposed TCM model in experiment II in terms of forecasting accuracy; the results of Wilcoxon signed-rank test in Table 5 shows that the hat the TCM have better performance than the single machine leaning models such as BPNN, SVM, and ELM. The levels of Wilcoxon signed-rank test between TCM and EEMD-SVM or TCM and EEMDELM are all smaller than the significance level 0.01, but the P value between TCM and EEMD-SVM is 0.653 , which means that the adaptability of TCM is not ideal, although in some 

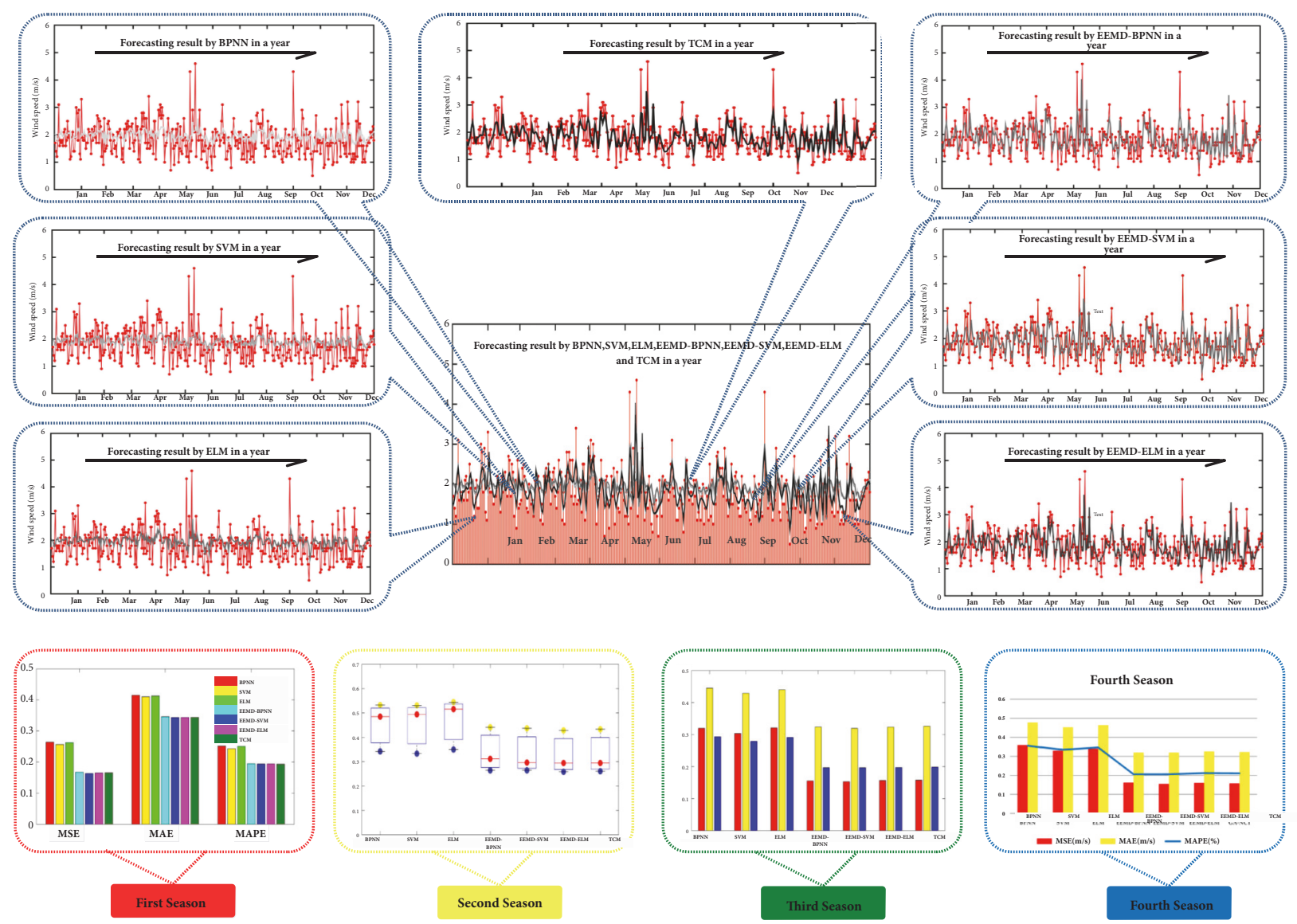

FIGURE 4: The forecasting performance of Experiment II for wind turbine II.

TABLE 3: The MSE, MAE and MAPE of experiment II for wind turbine II in each season.

\begin{tabular}{lcccccccc}
\hline Season & Evaluation criteria & BPNN & SVM & ELM & EEMD-BPNN & EEMD-SVM & EEMD-ELM & TCM \\
\hline \multirow{3}{*}{ First season } & MSE(m/s) & 0.2642 & 0.2569 & 0.2627 & 0.1676 & 0.1634 & 0.1652 & 0.1657 \\
& MAE(m/s) & 0.4149 & 0.4101 & 0.4131 & 0.3461 & 0.3435 & 0.3438 & 0.3441 \\
& MAPE(\%) & $25.18 \%$ & $24.33 \%$ & $25.06 \%$ & $19.51 \%$ & $19.40 \%$ & $19.42 \%$ & $19.34 \%$ \\
\hline \multirow{3}{*}{ Second season } & MSE(m/s) & 0.4851 & 0.4941 & 0.5156 & 0.3117 & 0.2966 & 0.2945 & 0.2949 \\
& MAE(m/s) & 0.5322 & 0.5309 & 0.5445 & 0.4414 & 0.4371 & 0.4283 & 0.4334 \\
& MAPE(\%) & $34.23 \%$ & $33.33 \%$ & $35.01 \%$ & $26.46 \%$ & $26.47 \%$ & $25.89 \%$ & $26.12 \%$ \\
\hline \multirow{3}{*}{ Third season } & MSE(m/s) & 0.3199 & 0.3034 & 0.321 & 0.1554 & 0.1529 & 0.1567 & 0.1577 \\
& MAE(m/s) & 0.445 & 0.4289 & 0.4399 & 0.3237 & 0.3193 & 0.3233 & 0.3260 \\
& MAPE(\%) & $29.33 \%$ & $27.90 \%$ & $29.11 \%$ & $19.65 \%$ & $19.64 \%$ & $19.71 \%$ & $19.85 \%$ \\
\hline \multirow{3}{*}{ Fourth season } & MSE(m/s) & 0.3599 & 0.3288 & 0.3399 & 0.1619 & 0.1555 & 0.1610 & 0.1585 \\
& MAE(m/s) & 0.4777 & 0.4527 & 0.4639 & 0.3195 & 0.3196 & 0.3254 & 0.3226 \\
& MAPE $(\%)$ & $35.55 \%$ & $33.46 \%$ & $34.68 \%$ & $20.57 \%$ & $20.65 \%$ & $21.18 \%$ & $21.08 \%$ \\
\hline
\end{tabular}

TABLE 4: Results of Wilcoxon signed-rank test for wind turbine I.

\begin{tabular}{lccccccc}
\hline Comparison & R+ & R- & P-value & Comparison & R+ & R- & P-value \\
\hline EEMD-BPNN vs. BPNN & 320 & 45 & 0.001 & TCM vs. EEMDBPNN & 359 & 6 & 0.001 \\
EEMD-SVM vs. SVM & 321 & 44 & 0.001 & TCM vs. EEMD-SVM & 353 & 12 & 0.001 \\
EEMD-ELM vs. ELM & 316 & 49 & 0.001 & TCM vs. EEMD-ELM & 315 & 50 & 0.001 \\
\hline
\end{tabular}


TABLE 5: Results of Wilcoxon signed-rank test wind turbine II.

\begin{tabular}{lccccccc}
\hline Comparison & R+ & R- & P-value & Comparison & R+ & R- & P-value \\
\hline TCM vs. BPNN & 258 & 107 & 0.001 & TCM vs. EEMD-BPNN & 170 & 195 & 0.653 \\
\hline TCM vs. SVM & 240 & 125 & 0.001 & TCM vs. EEMD-SVM & 242 & 123 \\
\hline TCM vs. ELM & 259 & 106 & 0.001 & TCM vs. EEMD-ELM & 217 & 0.001 \\
\hline
\end{tabular}

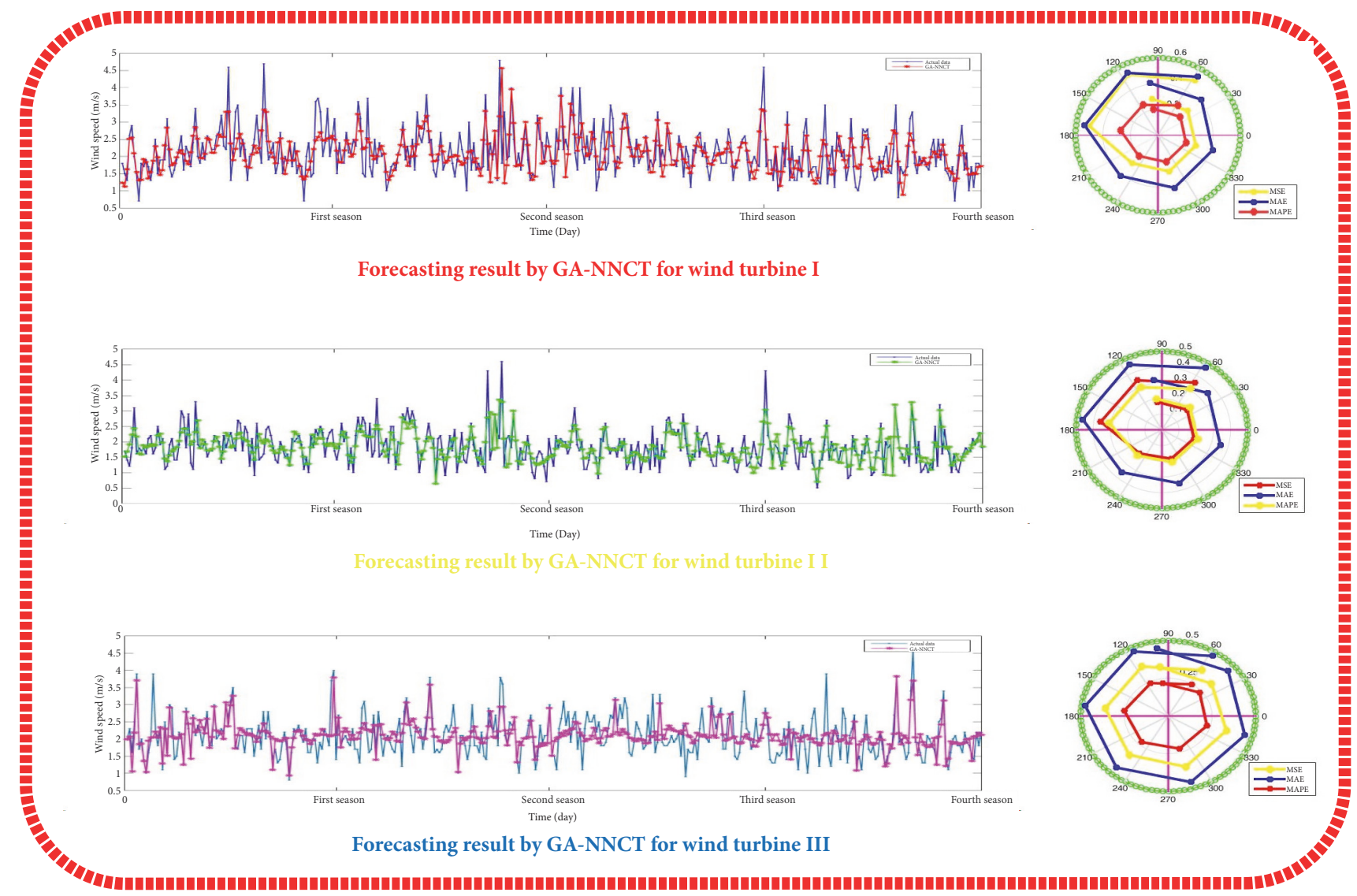

FIgURE 5: The forecasting performance of Experiment III for wind turbine I, wind turbine II, and wind turbine III.

cases it has obviously effect on improving the forecasting accuracy.

7.3. Experiment III: Daily Wind Speed Predicted by the GANNCT Model. The results of experiment II show that the TCM has weak adaptability and poor robustness, but wind speed is a key parameter in the wind energy system; how to improve the forecasting accuracy of wind speed forecasting models has an important effect on the wind generation. Usually, the forecasting of wind speed with higher precision is a challenging task. In this section, experiment III was designed to verify the forecasting performance of the ensemble GANNCT model using wind turbine I, wind turbine II, and wind turbine III datasets.

The experiment processes are designed as the follows.

Step 1. The GA-NNCT are used to forecast wind speed using wind turbine I and wind turbine II datasets.
Step 2. The models including BPNN, SVM, ELM, EEMDBPNN, EEMD-SVM, EMD-ELM, TCM, and GA-NNCT are used to forecast the wind speed using the wind turbine III dataset.

Step 3. The evaluation criteria of above models are compared in different time scale.

The MSE, MAE, and MAPE of BPNN, SVM, ELM, EEMD-BPNN, EEMD-SVM, EMD-ELM, TCM, and GANNCT are shown in Tables 6 and 7. The prediction results and errors of GA-NNCT in three wind turbines are shown in Figure 5. The MAPE is selected as the final benchmark and the main findings are listed below:

(a) All the three numerical experiment results illustrate that the advantage of ensemble model GA-NNCT is very obvious and the forecasting performance is superior to the best single machine leaning model in 


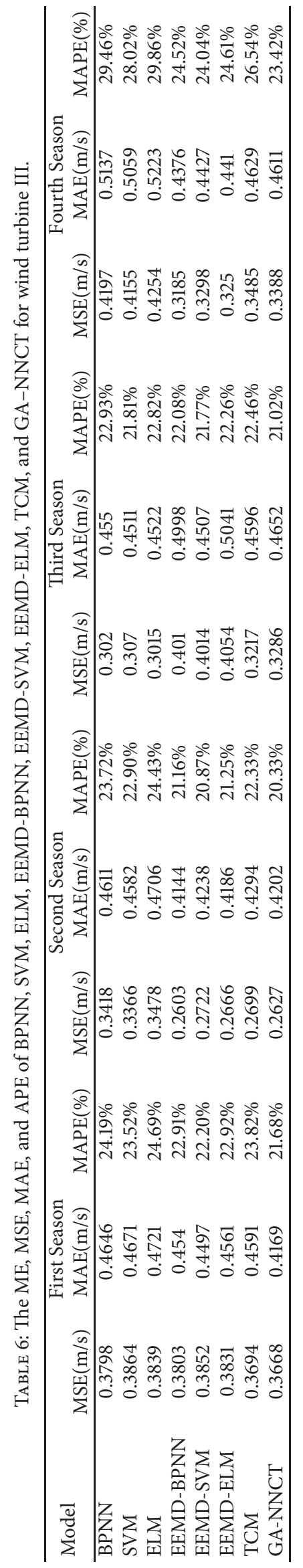




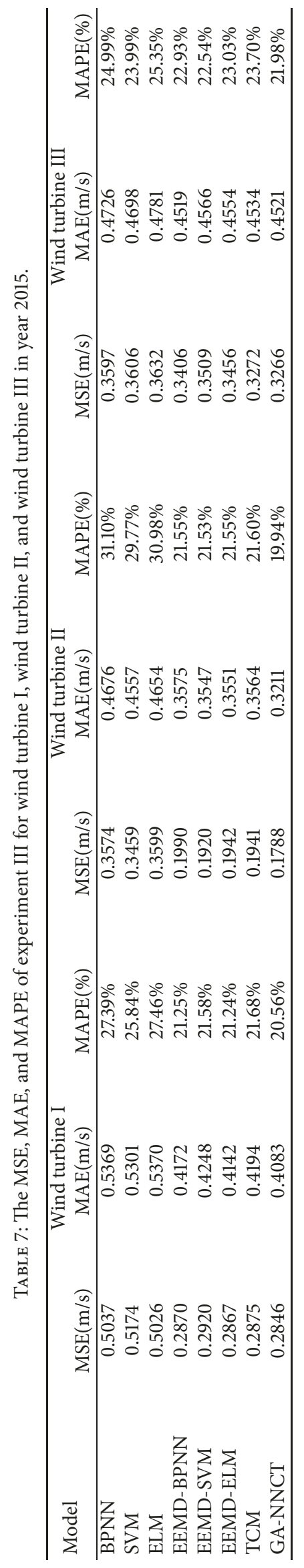


TABLE 8: Results of Wilcoxon signed-rank test wind turbine III.

\begin{tabular}{llcccccc}
\hline Comparison & R+ & R- & P-value & Comparison & R+ & R- & P-value \\
\hline GA-NNCT vs. BPNN & 213 & 152 & 0.001 & GA-NNCT vs. EEMD-BPNN & 339 & 26 & 0.001 \\
\hline GA-NNCT vs. SVM & 247 & 118 & 0.001 & GA-NNCT vs. EEMD-SVM & 331 & 34 \\
\hline GA-NNCT vs. ELM & 201 & 164 & 0.001 & GA-NNCT vs. EEMD-ELM & 198 & 167 \\
\hline
\end{tabular}

different time scale as shown in Tables 6 and 7. The calculation results show that some improvements of the predicting precision have been achieved.

(b) The MAPE of GA-NNCT are all smaller than the MAPE of EEMD-BPNN, EEMD-SVM, and EEMDELM in every time scale, which means that the combination GA-NNCT model has excellent forecasting performance among the six submodels in wind turbine I, wind turbine II, and wind turbine III.

(c) The MSE, MAE, and MAPE of TCM in wind turbine III prove again that the forecasting performance of TCM has weak adaptability and poor robustness in different time scale. Comparing with TCM, the MAPE of GA-NNCT are all smaller than TCM, which means that the ensemble model GA-NNCT performs much better than the TCM, which indicates that GANNCT has the higher adaptability and robustness in wind speed forecasting.

As shown in Table 6, the MAPE of EEMD-BPNN, EEMD-SVM, and EEMD-ELM are all smaller than BPNN, SVM, and ELM in each season, respectively, for wind turbine III in year 2015, and the decrease range of the MAPE is big in first season, second season, and fourth season; the range of MAPE decreased about $1.23 \%$ to $5.25 \%$. But in the fourth season, the MAPE of EEMD-BPNN, EEMD-SVM, and EEMD-ELM are decreased about $0.04 \%$ to $0.85 \%$, which indicate EEMD-BPNN, EEMD-SVM, and EEMD-ELM with a better accuracy compared to BPNN, SVM, and ELM in each season. In different seasons, the results in Table 6 show that the forecasting performance of TCM is no better than the hybrid models, even worse than the single machine leaning models sometimes. The MAPE of TCM are all greater than the hybrid models in each season, which indicate that the TCM has weak adaptability and robustness for wind speed forecasting in wind turbine III. Compared with the hybrid models and TCM, the advantage of ensemble model GA-NNCT is very obvious in each season; the forecasting performance is superior to the best hybrid model as shown in Table 6. The MAPE of GA-NNCT decreased about $0.52 \%$ to $3.12 \%$ in different seasons. In experiment III, the wind speed forecasting models which developed in this paper, the MAPE of GA-NNCT show that the prediction precision of the GANNCT is the highest and the hybrid models are in the second place. The prediction precision of TCM and single machine learning models need to be compared in different situations.

The MSE, MAE, and MAPE of BPNN, SVM, ELM, EEMD-BPNN, EEMD-SVM, EMD-ELM, TCM, and GANNCT for the wind turbine I, wind turbine II, and wind turbine III in year 2015 are shown in Table 7. The MAPE of GA-NNCT is $20.56 \%, 19.94 \%$, and $21.98 \%$ for wind turbine
I, wind turbine II, and wind turbine III, respectively, which are all smaller than the submodels and TCM. Compared to the submodels, the decrease range of the MAPE is $0.68 \%$ to $6.9 \%$ for wind turbine I in year 2015. As for wind turbine II and wind turbine III, the decrease range of the MAPE is $1.61 \%$ to $11.16 \%$ and $0.56 \%$ to $3.37 \%$, respectively, which indicate that the GA-NNCT model can forecast the daily wind speed with better forecasting performance in the Longdong area of Loess Plateau in China. The numerical results of experiment I, experiment II, and experiment III show that GA-NNCT has high robustness and adaptive ability.

Finally, the Wilcoxon signed-rank test is conducted to ensure the significant contribution in terms of forecasting accuracy improvement for the proposed GA-NNCT model. The test results in Table 8 show that the proposed GANNCT model almost reaches a significance level in terms of forecasting performance than other alternative compared models in experiment III, which means that GA-NNCT has high prediction accuracy and adaptive ability of wind speed forecasting.

\section{Conclusions}

Energy is always playing an important part in the development of national and regional economics. With the increasing depletion of fossil energy, the renewable energy development is raised rapidly in the various countries' energy development program. Wind energy becomes an important renewable and sustainable new resource due to the character of being environmentally friendly and reproducibility. However, wind speed is a key parameter in the wind energy system, and the prediction of wind speed is always a challenging issue; no single method or model can perform well in all situations; literature reviews show that the sole use of artificial intelligence model or the hybrid models based on data preprocessing technology and proper single model could not adequately forecast the wind speed with favorable performance. In this paper, three popular single models including BPNN, SVM, and ELM as well as three hybrid forecasting models based on data preprocessing including EEMD-BP, EEMD-SVM, and EEMD-ELM are used to forecast the daily average wind speed; the numerical results show that the accuracies of these models need to be further improved; then these submodels BPNN, SVM, ELM, EEMD-BP, EEMD-SVM, and EEMDELM are selected by the TCM and GA-NNCT, respectively; the wind speed is predicted using these ensemble models and the experimental data agreed with the model predictions. The numerical results show that the proposed combination model GA-NNCT has high prediction accuracy. This study can be a helpful tool for wind park management and conversion 
of wind power to electricity in the Longdong area of Loess Plateau in China.

\section{Data Availability}

The data used to support the findings of this study are available from the corresponding author upon request.

\section{Conflicts of Interest}

The authors declare that they have no conflicts of interest.

\section{Acknowledgments}

This work was supported by the Western Project of the National Social Science Foundation of China (Grant no. 18XTJ003).

\section{References}

[1] http://www.cehome.com/news/20160314/210615.shtml.

[2] I. Moghram and S. Rahman, "Analysis and evaluation of five short-term load forecasting techniques," IEEE Transactions on Power Systems, vol. 4, no. 4, pp. 1484-1491, 1989.

[3] A. Öztopal, "Artificial neural network approach to spatial estimation of wind velocity data," Energy Conversion and Management, vol. 47, no. 4, pp. 395-406, 2006.

[4] M. A. Mohandes, T. O. Halawani, S. Rehman, and A. A. Hussain, "Support vector machines for wind speed prediction," Journal of Renewable Energy, vol. 29, no. 6, pp. 939-947, 2004.

[5] R. E. Abdel-Aal, M. A. Elhadidy, and S. M. Shaahid, "Modeling and forecasting the mean hourly wind speed time series using GMDH-based abductive networks," Journal of Renewable Energy, vol. 34, no. 7, pp. 1686-1699, 2009.

[6] M. Bilgili, B. Sahin, and A. Yasar, "Application of artificial neural networks for the wind speed prediction of target station using reference stations data," Journal of Renewable Energy, vol. 32, no. 14, pp. 2350-2360, 2007.

[7] L. Kamal and Y. Z. Jafri, “Time series models to simulate and forecast hourly averaged wind speed inquetta, pakistan lalarukh kamal and yasmin zahra jafri," Solar Energy, vol. 61, no. 1, pp. 23-32, 1997.

[8] E. Cadenas and W. Rivera, "Short term wind speed forecasting in La Venta, Oaxaca, México, using artificial neural networks," Journal of Renewable Energy, vol. 34, no. 1, pp. 274-278, 2009.

[9] Y. Dong, Z. Guo, J. Wang, and H. Lu, “The forecasting procedure for long-term wind speed in the Zhangye area," Mathematical Problems in Engineering, vol. 2010, Article ID 684742, 17 pages, 2010.

[10] O. B. Shukur and M. H. Lee, "Daily wind speed forecasting through hybrid KF-ANN model based on ARIMA," Journal of Renewable Energy, vol. 76, pp. 637-647, 2015.

[11] J. M. Hu, J. Z. Wang, and G. W. Zeng, "A hybrid forecasting approach applied to wind speed time series," Journal of Renewable Energy, vol. 60, pp. 185-194, 2013.

[12] Z. H. Guo, J. Zhao, W. Y. Zhang, and J. Z. Wang, "A corrected hybrid approach for wind speed prediction in Hexi Corridor of China," Energy, vol. 36, no. 3, pp. 1668-1679, 2011.
[13] J. Wang, W. Zhang, J. Wang, T. Han, and L. Kong, "A novel hybrid approach for wind speed prediction," Information Sciences, vol. 273, pp. 304-318, 2014.

[14] J. Shi, J. Guo, and S. Zheng, "Evaluation of hybrid forecasting approaches for wind speed and power generation time series," Renewable \& Sustainable Energy Reviews, vol. 16, no. 5, pp. 34713480, 2012.

[15] J. Wang, Y. Song, F. Liu, and R. Hou, "Analysis and application of forecasting models in wind power integration: a review of multi-step-ahead wind speed forecasting models," Renewable of Sustainable Energy Reviews, vol. 60, pp. 960-981, 2016.

[16] W. G. Zhao, J. Z. Wang, and H. Y. Lu, "Combining forecasts of electricity consumption in China with time-varying weights updated by a high-order Markov chain model," Omega , vol. 45, pp. 80-91, 2014.

[17] L. Xiao, J. Wang, Y. Dong, and J. Wu, "Combined forecasting models for wind energy forecasting: A case study in China," Renewable \& Sustainable Energy Reviews, vol. 44, pp. 271-288, 2015.

[18] P. Jiang and X. Ma, "A hybrid forecasting approach applied in the electrical power system based on data preprocessing, optimization and artificial intelligence algorithms," Applied Mathematical Modelling: Simulation and Computation for Engineering and Environmental Systems, vol. 40, no. 23-24, pp. 10631-10649, 2016.

[19] J. Che, "Optimal sub-models selection algorithm for combination forecasting model," Neurocomputing, vol. 151, no. 1, pp.364375, 2015.

[20] X. Ma and D. Liu, "Comparative Study of Hybrid Models Based on a Series of Optimization Algorithms and Their Application in Energy System Forecasting," Energies, vol. 9, no. 8, p. 640, 2016.

[21] G. Santamaría-Bonfil, A. Reyes-Ballesteros, and C. Gershenson, "Wind speed forecasting for wind farms: a method based on support vector regression," Journal of Renewable Energy, vol. 85, pp. 790-809, 2016.

[22] N. E. Huang, Z. Shen, S. R. Long et al., “The empirical mode decomposition and the Hilbert spectrum for nonlinear and non-stationary time series analysis," Proceedings A, vol. 454, pp. 903-995, 1998.

[23] Z. Wu and N. E. Huang, "Ensemble empirical mode decomposition: a noise-assisted data analysis method, centre for oceanland-atmosphere studies,” Tech Rep 193(51), 2004.

[24] S. Li, L. Goel, and P. Wang, "An ensemble approach for shortterm load forecasting by extreme learning machine," Applied Energy, vol. 170, pp. 22-29, 2016.

[25] M. Leuenberger and M. Kanevski, "Extreme Learning Machines for spatial environmental data," Computers \& Geosciences, vol. 85, pp. 64-73, 2015.

[26] X.-S. Yang and S. Deb, "Engineering optimisation by Cuckoo search," International Journal of Mathematical Modelling and Numerical Optimisation, vol. 1, no. 4, pp. 330-343, 2010.

[27] S. M. Homayouni, S. H. Tang, and O. Motlagh, "A genetic algorithm for optimization of integrated scheduling of cranes, vehicles, and storage platforms at automated container terminals," Journal of Computational and Applied Mathematics, vol. 270, pp. 545-556, 2014.

[28] http://baike.so.com/doc/5346514-5581961.html.

[29] J. Derrac, S. García, D. Molina, and F. Herrera, "A practical tutorial on the use of nonparametric statistical tests as a 
methodology for comparing evolutionary and swarm intelligence algorithms," Swarm and Evolutionary Computation, vol. 1, no. 1, pp. 3-18, 2011.

[30] Y. Dong, Z. Zhang, and W. Hong, "A Hybrid Seasonal Mechanism with a Chaotic Cuckoo Search Algorithm with a Support Vector Regression Model for Electric Load Forecasting," Energies, vol. 11, no. 4, p. 1009, 2018. 


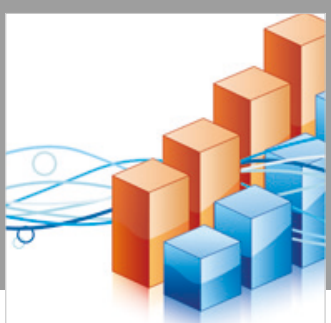

Advances in

Operations Research

\section{-n-m}
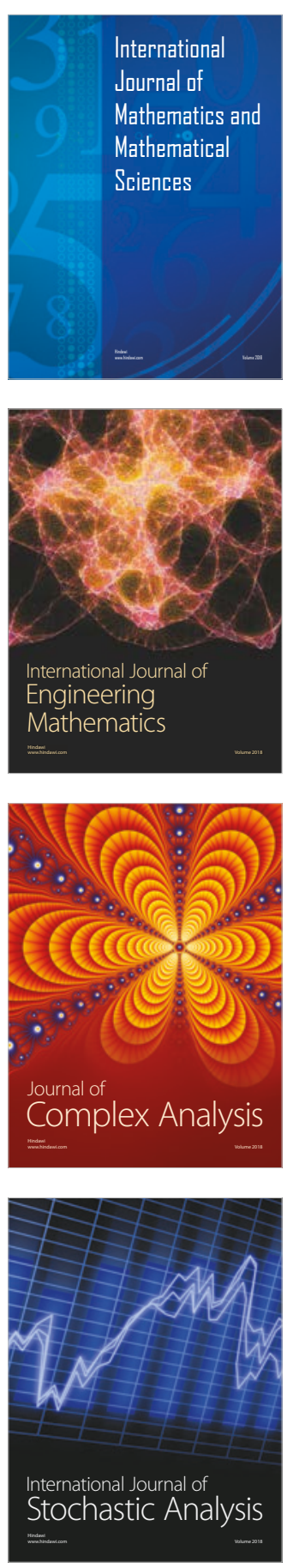
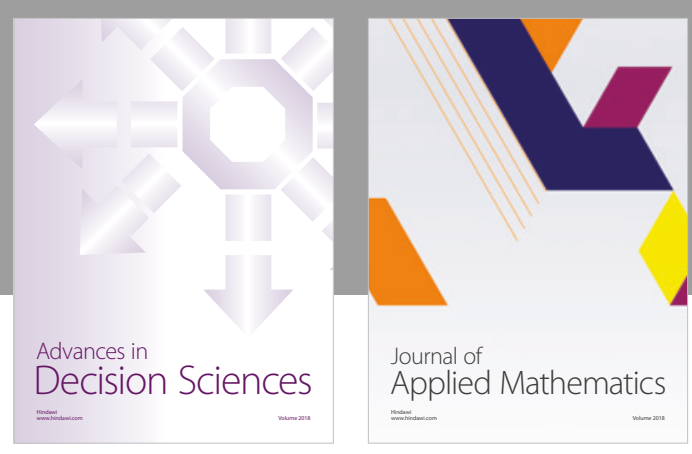

Journal of

Applied Mathematics


Submit your manuscripts at

www.hindawi.com



Advances in
Numerical Analysis


Mathematical Problems in Engineering



International Journal of

Differential Equations

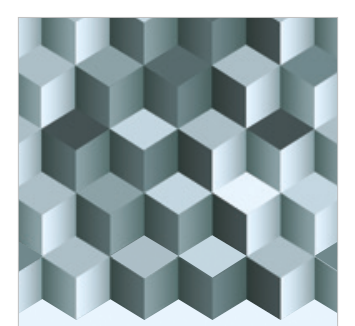

Journal of

Function Spaces



The Scientific

World Journal



Journal of

Probability and Statistics
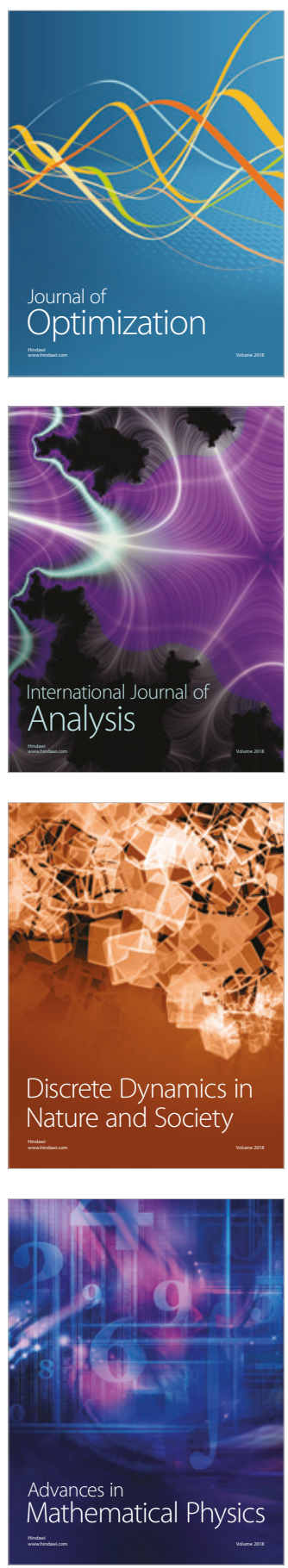